\title{
Proof of Concept Study to Investigate the Association Between Autovaccines and COVID-19 Incidence in Patients with Recurrent Respiratory Diseases
}

\author{
Jaime Leache Pueyo ${ }^{1}$, Laura Pérez Delgado ${ }^{1}$, José Victor Tisner Nieto ${ }^{2}$, Ma Carmen Orte Aldea ${ }^{3}$, \\ Raquel Artal Sánchez ${ }^{2}$, María Matas $\operatorname{Ros}^{4}$ and Inmaculada Buendía Jiménez ${ }^{4 *}$ \\ ${ }^{1}$ Hospital Universitario Miguel Servet, ENT Service, Zaragoza, Spain
}

${ }^{2}$ ENT Clinic, Zaragoza, Spain

${ }^{3}$ ENT Service, Huesca, Spain

${ }^{4}$ Probelte Pharma S.L.U, Murcia, Spain

*Corresponding author: Inmaculada Buendía Jiménez, Probelte Pharma S.L.U, Murcia, Spain

\section{ARTICLE INFO}

Received: 画 June 01, 2021

Published: 幽 June 11, 2021

Citation: Jaime Leache Pueyo, Laura Pérez Delgado, José Victor Tisner Nieto, Ma ${ }^{\mathrm{a}}$ Carmen Orte Aldea, Inmaculada Buendía Jiménez, et al., Proof of Concept Study to Investigate the Association Between $\mathrm{Au}$ tovaccines and COVID-19 Incidence in Patients with Recurrent Respiratory Diseases. Biomed J Sci \& Tech Res 36(3)-2021. BJSTR. MS.ID.005853.

Keywords: Autovaccine; Bacterial Lysate; COVID-19; Trained Innate Immunity

\section{ABSTRACT}

Introduction: Exposure to bacterial components can activate innate immunity and "train" it.

This phenomenon is crucial in the fight against infectious diseases, including COVID-19. The objective of this study is to assess whether a bacterial autovaccine could protect against SARS-CoV-2 thanks to trained innate immunity.

Methods: A retrospective controlled study that was carried out in four ENT services in Spain. Patients aged between 18 and 80 years of age with recurrent upper respiratory tract infections who received the bacterial autovaccine during a period between January 2019 and April 2020 were selected. The control group was made up of a selection of patients who presented a pathology in the ENT area and who did not receive the bacterial autovaccine during the same period. The study was approved by the regional Ethics Committee of Aragon. The main parameter was cumulative incidence, i.e., the proportion of people who contracted COVID-19 and had been diagnosed by PCR or serology in the period between March and August 2020 in each group.

Results: 129 patients were included in the autovaccine group and 149 patients in the control group. Both groups had a similar demographic and clinical distribution. The group treated with the autovaccine had a significantly lower incidence of COVID-19 than the control group $(2.3 \%$ vs $8.7 \%, \mathrm{p}<0.05)$.

Conclusion: These results suggest that the bacterial autovaccine could protect against COVID-19 as the cumulative incidence in the autovaccine group is almost four times lower than in the control group. Controlled clinical trials are necessary to confirm these data.

\section{Introduction}

Bacterial lysate vaccines are indicated for the prevention of recurrent respiratory infections in adults and children [1-3]. This treatment enhances the adaptative response against the specific pathogens contained in the vaccine. Additionally, it also boosts the baseline tone of innate immunity by developing immunological long-term memory and improving the cross protection from other pathogens $[4,5]$. This process is known as trained immunity and may have an important role in the fight against infectious diseases, 
including COVID-19 [6-8]. On the basis of this data, we hypothesized that the bacterial autovaccines may be a preventive measure against SARS-CoV-2.

\section{Materials and Methods}

We did a multicenter retrospective case-control study of data from the ENT department at 3 hospitals and one clinic in the region of Aragon (Spain) to estimate the autovaccine effectiveness against the COVID-19. Our study population included people aged between 18 and 80 years of age who were diagnosed with recurrent upper respiratory tract infections at the participating medical sites, and who had received the autovaccine (Probelte Pharma, Murcia, Spain) during a period between January 2019 and April 2020. The control group consisted of a random selection of patients diagnosed with a chronic ENT pathology during the same period at the investigator sites. Data sources were the medical history, which included COVID-19 diagnoses. This has been routinely recorded in the patient medical history at regular medical consultations since the beginning of the pandemic in March 2020. The data about the COVID-19 infection was provided for the period between March and August 2020. We obtained ethical approval from the regional Ethics Committee in Aragon (CEICA). The autovaccine is a bacterial lysate from bacterial germens isolated from the respiratory mucosa of each patient. The vaccine was sublingually administered daily for at least 3 months before the COVID-19 diagnostic test. The primary outcome of the study was the cumulative incidence, i.e., the proportion of people who contracted the COVID-19 disease and had

Table 1: Baseline characteristics of the population. been diagnosed by a PCR or serology test, in the period between March and August 2020 in each study group. The secondary outcomes were the proportion of patients hospitalized and in intensive care units because of the infection. Data were reported as means and standard deviations after checking for normality of distribution. Paired t-tests for independent groups and Chi-square tests were used to compare values between the groups. The study was not statistically powered given the exploratory design and the absence of previous literature on the topic.

\section{Results}

A total of 140 patients received the autovaccine at the participant investigator sites during the study period. Finally, 129 patients met the inclusion criteria in the autovaccine group and 149 patients in the control group (Figure 1). Overall, baseline demographic and clinical parameters were similar in treated and control patients Table 1. The mean (SD) age of all patients was 52 (17) years, and the mean body mass index (SD) was 26 [6]. The majority of patients received a mixture of two bacteria and $80 \%$ of them contained Streptococcus species, including pneumoniae, viridans and pyogenes (Figure 2). The autovaccine group had a significant lower cumulative incidence of COVID-19 compared with the control group. The proportion of patients who contracted COVID-19 in the treated group was $2.3 \%$ and, in the control, group was $8.7 \%$, meaning a difference between groups of $74 \%(\mathrm{p}<0.05)$. The proportion of patients hospitalized and in ICUs was also higher in the control group but did not reach a statistical difference.

\begin{tabular}{|c|c|c|c|}
\hline Variable & Autovaccine Group & Control Group & P-Value \\
\hline Age (years) - Mean (SD) & $49.7(18)$ & $54.1(16)$ & $\mathrm{p}>0.05$ \\
\hline BMI -Mean (SD) & $25.0(4)$ & $26.3(7)$ & $\mathrm{p}>0.05$ \\
\hline Gender -Female. \% & 67.4 & 52.0 & $\mathrm{P}<0.05^{*}$ \\
\hline Race -Caucasian. \% & 93.7 & 97.0 & $\mathrm{p}>0.05$ \\
\hline Smoker. \% & 14.7 & 17.0 & $\mathrm{p}>0.05$ \\
\hline CV Disease. \% & 14.7 & 21.5 & $\mathrm{p}>0.05$ \\
\hline Diabetes. \% & 7.0 & 6.1 & $\mathrm{p}>0.05$ \\
\hline Lung disease & 7.8 & 7.5 & $\mathrm{P}>0.05$ \\
\hline Immunodepression. \% & 3.9 & 3.4 & $\mathrm{P}>0.05$ \\
\hline Autoimmune disease. \% & 9.4 & 3.4 & $\mathrm{p}>0.05$ \\
\hline Neurodegenerative disease. $\%$ & 0.8 & 0.7 & $\mathrm{p}>0.05$ \\
\hline Hepatic impairment. $\%$ & 0 & 2.1 & $\mathrm{p}>0.05$ \\
\hline Renal impairment. $\%$ & 0 & 6.8 & $\mathrm{P}<0.05^{*}$ \\
\hline Cancer. $\%$ & 1.6 & 7.5 & $\mathrm{P}<0.05^{*}$ \\
\hline
\end{tabular}




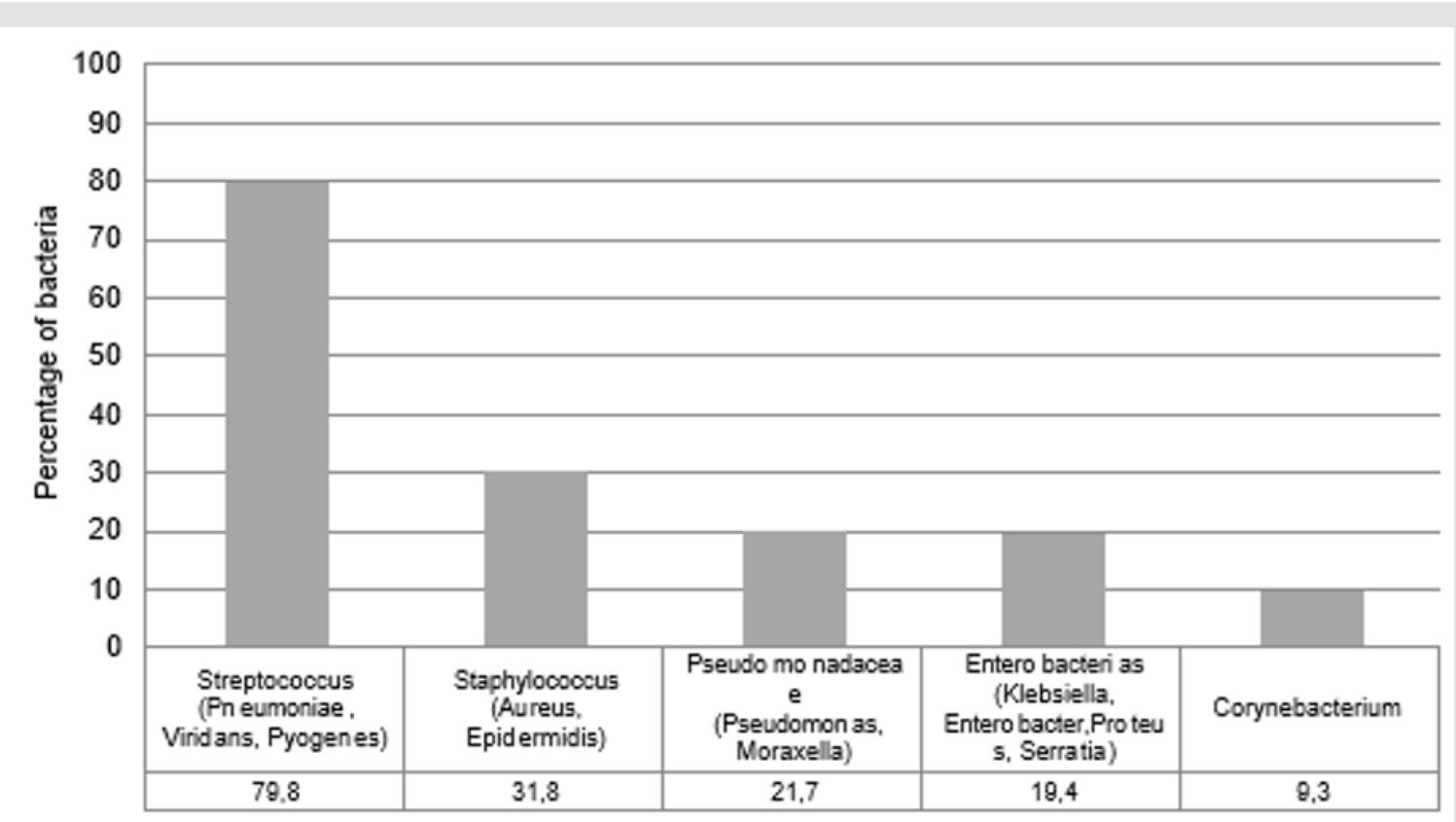

Figure1: Autovaccine bacterial composition.

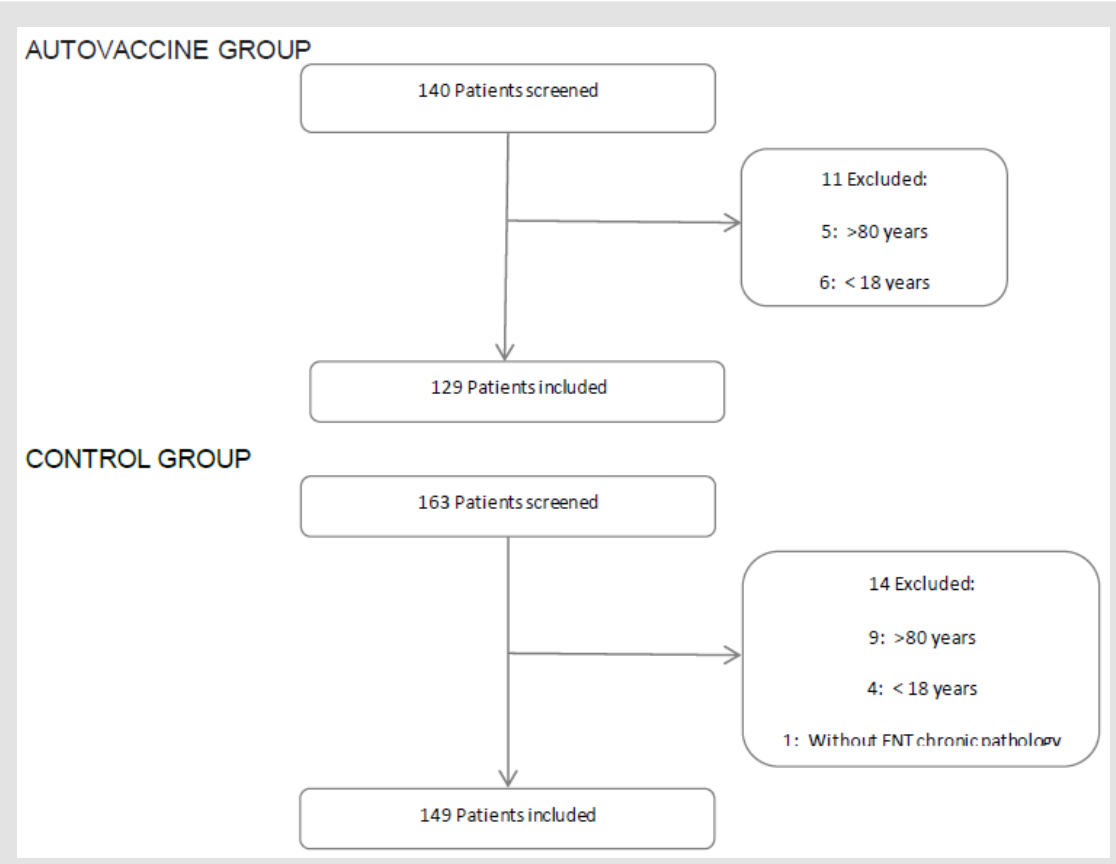

Figure 2: Study flow chart.

\section{Discussion}

This data suggests that the autovaccine may protect against COVID-19 since the cumulative incidence in the treated group in almost four times lower than in the control group. As with any observational study, this analysis has some limitations. The results may be influenced by a limited sample size and slight differences between the groups at baseline. The control group had a lower proportion of males and a slightly higher prevalence of cancer and chronic kidney disease compared to the autovaccine group. However, previous studies have reported that this may not impact the incidence of COVID-19 [9,10]. Conversely, the study has several strengths. The study population is representative of the general population, the COVID-19 diagnosis was confirmed by a physician and a rigorous procedure was followed to obtain a random selection of control patients from the appointment list at each site. At present, it is crucial to explore new options to control the worldwide COVID-19 pandemic. A number of conventional 
anti infection vaccines with heterologous protection are under investigation. About 200 hundred studies have been published to assess the effectiveness of the anti-tuberculosis (BCG) vaccine against COVID-19. The results remain controversial and several clinical trials are under way. The bacterial lysates vaccines from respiratory pathogens can also train immunity to more efficiently fight heterogeneous pathogens. Additionally, some pathogens such as Streptococcus pneumoniae, included in the autovaccines administered in the study, may have a potential cross reactivity immunity to SARS-COV-2, as described by Roche et al. [11]. However, apparently no study has yet assessed the protective role of the autovaccine or bacterial lysate to fight against the SARSCoV-2. Our study, although preliminary, suggests the autovaccine as a possible prophylactic treatment that may contribute to the fight against COVID-19. Larger cohorts and randomized controlled trials are urgently needed to confirm these results.

\section{Funding}

No funding was received for this work.

\section{Conf Lists of Interest}

Jaime Leache Pueyo has no conflict of interest. Laura Pérez Delgado has no conflict of interest. José Victor Tisner Nieto has no conflict of interest. Ma Carmen Orte Aldea has no conflict of interest. Raquel Artal Sánchez has no conflict of interest.

María Matas Ros is an employee of Probelte Pharma.

Inmaculada Buendía Jiménez is an employee of Probelte Pharma.

\section{References}

1. Cazzola M, Anapurapu S, Page CP (2012) Polyvalent mechanical bacterial lysate for theprevention of recurrent respiratory infections: A metaanalysis. Pulmonary Pharmacology \&Therapeutics. 25(1): 62-68.

\section{ISSN: 2574-1241}

\section{DOI: 10.26717/BJSTR.2021.36.005853}

Inmaculada Buendía Jiménez. Biomed J Sci \& Tech Res

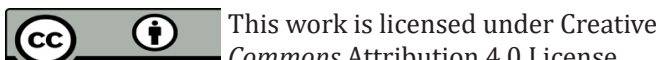

Submission Link: https://biomedres.us/submit-manuscript.php
2. Del-Rio-Navarro BE, Espinosa Rosales F, Flenady V, et al. (2006) Immunostimulants for preventing respiratory tract infection in children. Cochrane Database Syst Rev. 18(4): CD004974.

3. Yin J, Xu B, Zeng X, Kunling Shen (2018) Broncho-Vaxom in pediatric recurrent respiratory tractinfections: a systematic review and metaanalysis. Int Immunopharmacol 54: 198-209.

4. Bellanti JA, Settipane RA (2009) Bacterial vaccines and the innate immune system: A journeyof rediscovery for the allergist-immunologist and all health care providers. Allergy Asthma Proc. 30: S3-S4.

5. Sanchez-Ramon S, Conejero L, Netea MG, Sancho D, Palomares O, et al. (2018) Trainedimmunity-based vaccines: a new paradigm for the development of broad-spectrum anti-infectious formulations. Frontiers in immunology 9: 2936.6 .

6. Mantovani A, Netea MG (2020) Trained innate immunity, epigenetics, and COVID-19. The NewEngland Journal of Medicine 383: 11.

7. Netea MG, Giamarellos-Bourboulis EJ, Dominguez-Andrés J, Curtis $\mathrm{N}$, Van Crevel R, et al. (2020) Trained immunity: a tool for reducing susceptibility to and the severity of SARS-CoV-2 infection. Cell. 181(5): 969-977.

8. Larenas-Linnemann D, Rodriguez-Pérez N, Arias-Cruz A, BlandónVijil MV, Del Río-Navarro BE, et al. (2020) Enhancing innate immunity against virus in times of COVID-19: trying to untangle facts from fictions. WAO Journal 13 (11): 100476.

9. Pollán M, Pérez-Gómez B, Pastor-Barriuso R, Oteo J, Hernán MA, et al. (2020) Prevalence of SARS-CoV-2 in Spain (ENE-COVID): a nationwide, population-basedseroepidemiological study. Lancet. 396: 535-544.

10. Uzzo R G, Kutikov A, Geynisman D (2019) Coronavirus disease 2019: risk for infection, clinical presentation, testing and management in patients with cancer.

11. Reche PA (2020) Potential cross-reactive immunity to SARS-CoV-2 from common human pathogens and vaccines. Frontiers in immunology 11: 586984 .

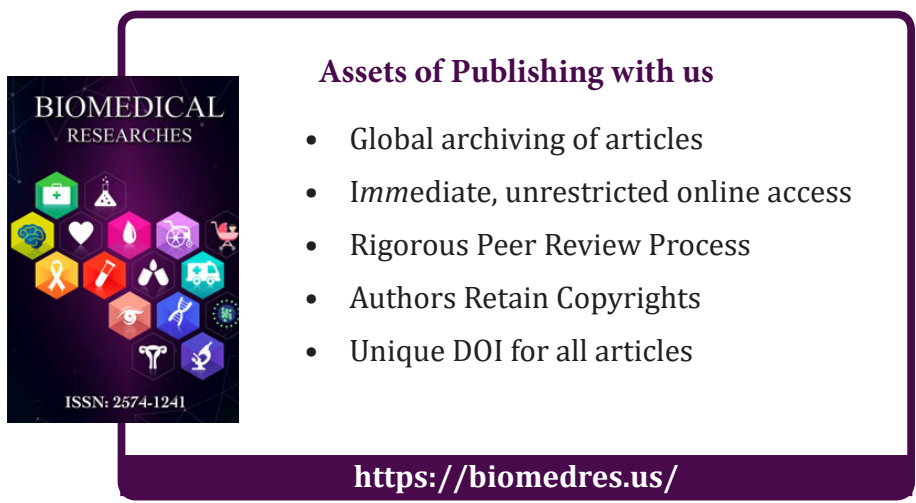

Copyright@ Inmaculada Buendía Jiménez | Biomed J Sci \& Tech Res | BJSTR. MS.ID.005853. 